\title{
Interventional Potential of Recombinant Feline Hepatocyte Growth Factor in a Mouse Model of Non-alcoholic Steatohepatitis
}

\author{
Yoon Mee Yang ${ }^{1+}$, Masato Fukui ${ }^{2+}$, Zhijun Wang ${ }^{1}$, Fiona Miao ${ }^{1}$, Margo J. Karriker ${ }^{2}$ and \\ Ekihiro Seki ${ }^{1,3,4,5 *}$ \\ 1 Division of Digestive and Liver Diseases, Department of Medicine, Cedars-Sinai Medical Center, Los Angeles, CA, \\ United States, ${ }^{2}$ Veterinary Medical Center-San Diego, University of California, San Diego, San Diego, CA, United States, \\ ${ }^{3}$ Department of Biomedical Sciences, Cedars-Sinai Medical Center, Los Angeles, CA, United States, ${ }^{4}$ Division of \\ Gastroenterology, Department of Medicine, School of Medicine, University of California, San Diego, La Jolla, CA, \\ United States, ${ }^{5}$ Department of Medicine, David Geffen School of Medicine, University of California, Los Angeles, Los \\ Angeles, CA, United States
}

OPEN ACCESS

Edited by:

Yong-ho Lee,

College of Medicine, Yonsei University,

South Korea

Reviewed by:

Kenichiro Mikami,

Hirosaki University, Japan

Naoya Yamada,

Jichi Medical University, Japan

Hyun Min Kim

Chung-Ang University, South Korea

*Correspondence:

Ekihiro Seki

ekihiro.seki@cshs.org

tThese authors have contributed equally to this work.

Specialty section: This article was submitted to

Cellular Endocrinology, a section of the journal Frontiers in Endocrinology

Received: 27 March 2018 Accepted: 22 June 2018

Published: 23 July 2018

Citation:

Yang YM, Fukui M, Wang Z, Miao F, Karriker MJ and Seki E (2018)

Interventional Potential of Recombinant Feline Hepatocyte Growth Factor in a Mouse Model of

Non-alcoholic Steatohepatitis.

Front. Endocrinol. 9:378.

doi: 10.3389/fendo.2018.00378
Background and Aims: Hepatocyte growth factor (HGF) is a multifunctional pleiotropic protein involved in tissue regeneration, protection, angiogenesis, anti-inflammatory and anti-fibrotic responses, and tumorigenesis, through binding to its receptor MET. Recombinant HGF protein has been shown to mitigate various liver disease models, such as alcohol-induced liver injury, hepatic ischemia-reperfusion injury, and fibrosis. This study aimed to investigate the anti-inflammatory, anti-fibrotic, and anti-lipogenic effects of exogenous administration of feline HGF on a non-alcoholic steatohepatitis (NASH) mouse model.

Methods: Wild-type C57BL/6 mice were fed a choline-deficient amino acid defined (CDAA) diet for 3 weeks to create the mouse model of $\mathrm{NASH}$, which displays hepatic steatosis, inflammation, injury, and very mild fibrosis. One $\mathrm{mg} / \mathrm{kg}$ of recombinant feline HGF was administered intravenously daily in the last 7 days of the total 3 weeks of CDAA diet feeding. Then, hepatic steatosis, inflammation, injury, and fibrogenic gene expression was examined.

Results: After 3 weeks of a CDAA diet-feeding, the vehicle-treated mice exhibited evident deposition of lipid droplets in hepatocytes, inflammatory cell infiltration, and hepatocyte ballooning along with increased serum ALT levels whereas recombinant HGF-treated mice showed reduced hepatic steatosis, inflammation, and ballooned hepatocytes with a reduction of serum ALT levels. Recombinant HGF administration promoted hepatocyte proliferation. Increased hepatic lipid accumulation was accompanied by elevated expression of lipogenesis genes Fasn and Dgat1 in vehicle-treated mice. In HGF-treated mice, these genes were reduced with a decrease of lipid accumulation in the liver. Consistent with the anti-inflammatory property of HGF, augmented macrophage infiltration and upregulation of chemokines, Cxcl1, Ccl2, and Ccl5 in the CDAA diet fed mice, were suppressed by the addition of the HGF treatment. Finally, we examined the fibrotic response. The vehicle-treated mice had mild fibrosis with upregulation of Col1a1, Acta2, 
Timp1, Tgfb1, and Serpine1 expression. Recombinant HGF treatment significantly suppressed fibrogenic gene expression and collagen deposition in the liver.

Conclusion: Recombinant feline HGF treatment suppressed the progression of $\mathrm{NASH}$ in a CDAA diet feeding mouse model. This suggests that recombinant HGF protein has therapeutic potential for $\mathrm{NASH}$.

Keywords: HGF, NAFLD, NASH, recombinant, inflammation

\section{INTRODUCTION}

Non-alcoholic fatty liver disease (NAFLD) is a hepatic manifestation of metabolic syndrome, which is characterized by excessive accumulation of fat content in hepatocytes. The development of NAFLD is highly associated with obesity and type 2 diabetes. Currently, 25\% of adults suffer from NAFLD in the United States. NAFLD encompasses two clinicopathological entities, simple steatosis and non-alcoholic steatohepatitis (NASH). NASH is histologically characterized by hepatic steatosis along with inflammatory cell infiltration, ballooned hepatocytes, and pericellular, "chicken wire," fibrosis (1-4). $\mathrm{NASH}$-induced fibrosis may further progress to cirrhosis, and up to $13 \%$ of NASH patients with cirrhosis eventually develop hepatocellular carcinoma (HCC) (1-5). Currently, NASH-mediated cirrhosis is the second leading cause of liver transplantation, and it is predicted that this disease will be the leading cause of liver transplantation within the next 10 years (5). To date, lifestyle intervention, including weight loss and exercise, is the primary treatment for NAFLD, and there is no effective preventive or therapeutic drugs for NAFLD. Since there is a chronic shortage of liver donors, an unmet need for new and effective therapies for NAFLD is significant.

Not only in the human healthcare field, but also in veterinary medicine field, liver disease is a significant health concern in canines and felines (6). Hepatic lipidosis, which shares similar histological morphologies with human NAFLD, developed in the elderly, overweight cats that lost weight after not eating for days to weeks (6). This condition often leads to unfavorable clinical outcomes. Similar to that of human NAFLD, there is also a significant unmet need for effective therapies for fatty liver disease in animals.

Hepatocyte growth factor (HGF) is a multifunctional pleiotropic cytokine. HGF is initially produced as a biologically inactive, single-chain precursor form (7). After proteolytic cleavage, pro-HGF converts to the active form consisting of heterodimeric $69 \mathrm{kDa} \alpha$-chain and $34 \mathrm{kDa} \beta$-chain (7). The active form of HGF promotes tissue regeneration, protection, wound healing, angiogenesis, tumorigenesis, and anti-inflammatory, anti-apoptotic, and anti-fibrotic responses

\footnotetext{
Abbreviations: ALT, alanine aminotransferase; CDAA, choline-deficient amino acid defined; CSAA, choline-supplemented amino acid defined diet; HCC, hepatocellular carcinoma; H\&E, hematoxylin and eosin; HCC, hepatocellular carcinoma; HGF, hepatocyte growth factor; MMP, matrix metalloprotease; NAFLD, non-alcoholic fatty liver disease; NASH, non-alcoholic steatohepatitis; PCNA, proliferating cellular nuclear antigen; PCR, polymerase chain reaction; SMA, smooth muscle actin.
}

in a wide variety of organs, through binding to its receptor MET. In the liver, HGF plays a crucial role in liver regeneration after hepatectomy or massive liver damage, protection against hepatocyte apoptosis and necrosis, and suppression of liver fibrosis progression (7). Previous studies reported that administration of recombinant HGF mitigated alcohol-induced liver damage, ischemia-reperfusion liver injury, endotoxin-induced fulminant hepatitis, and liver fibrosis (812). The studies using transgenic mice overexpressing $\mathrm{Hgf}$ and hepatocyte-specific $c$-met knockout mice, indicate the protective role of the HGF-MET pathway in the development of NAFLD $(13,14)$. Although there is a report with the application using HGF gene therapy on a rat model of NAFLD-fibrosis (15), to the best of our knowledge, the therapeutic effect of recombinant HGF protein on the development of NASH has not been reported.

The high-fat diet-induced fatty liver model does not develop liver injury, inflammation, and fibrosis in a short period of feeding. The feeding of methionine-choline-deficient diet, commonly used for NASH preclinical studies, significantly reduces their body weight. These models do not well-recapitulate the pathophysiology of human NAFLD. Notably, rodents fed a choline-deficient amino acid-defined (CDAA) diet for 3 weeks develop hepatic steatosis, inflammation, and mild liver fibrosis without reducing body weight $(16,17)$. Therefore, we decided to use CDAA diet feeding to develop a mouse model of NASH in this study.

The primary purpose of this study is to investigate the therapeutic effect of recombinant HGF protein on the progression of NASH. Since the amino acid sequence of feline HGF shows 97.5, 93.3, and 93.2\% homology with those of canine, mouse, and human $(18,19)$, the second aim of this study is that to examine whether feline-derived recombinant HGF can be used for the treatment of animals with liver diseases using a mouse model of NASH.

\section{MATERIALS AND METHODS}

\section{Animal Experiments}

Animal experiments were performed in accordance with National Institutes of Health recommendations outlined in the Guide for the Care and Use of Laboratory Animals. All animal experiment protocols were approved by the University of California San Diego Institutional Animal Care and Use Committee. Male C57BL/6 mice were purchased from The Jackson Laboratory (Bar Harbor, MA) and were maintained in a $12 \mathrm{~h}$ light/dark cycle. Mice at 8 weeks of age were subjected to 
feeding ad libitum either choline-supplemented L-amino aciddefined diet (CSAA; catalog \#518754; Dyets Inc, Bethlehem, PA) or choline-deficient L-amino acid-defined diet (CDAA; catalog \#518753; Dyets Inc) for 3 weeks. Vehicle or $1 \mathrm{mg} / \mathrm{kg}$ of recombinant feline HGF was intravenously injected daily during the last 7 days.

\section{Histologic Examination}

Mouse liver tissues were fixed in 10\% neutral buffered formalin phosphate (Fisher Scientific, Pittsburgh, PA) and then embedded into paraffin blocks. $5-\mu \mathrm{m}$ thick sections were cut on a microtome (Thermo Scientific, Waltham, MA). Tissues were stained with hematoxylin and eosin for the evaluation of NAFLD Activity Score (steatosis, lobular inflammation, hepatocyte ballooning) and fibrosis as described (20). Immunochemistry for proliferating cellular nuclear antigen (PCNA), F4/80 and Sirius Red staining were performed as previously reported (21, 22). In brief, liver sections were incubated with monoclonal antibody to PCNA (clone PC10; Biolegend, San Diego, CA) using the MOM kit (Vector Laboratories, Burlingame, CA). Sections were incubated with monoclonal antibody to F4/80 (clone BM8; eBioscience, San Diego, CA) for immunohistochemical analysis of F4/80 expression or incubated with a solution of saturated picric acid containing 0.1\% Fast Green FCF (Sigma-Aldrich, St Louis, MO) and 0.1\% Direct Red 80 (Sirius Red R3B; SigmaAldrich, St Louis, MO) for Sirius Red staining. For Oil Red O staining, mouse liver tissues were fixed in $4 \%$ neutral buffered formalin phosphate and then embedded into OCT compound. Frozen liver tissues were sliced into 5- $\mu \mathrm{m}$ sections and stained with Oil Red O. PCNA or F4/80 or Oil Red O-positive area was evaluated from randomly selected 10 fields of x 200 magnification per slide and quantified with NIH Image J software.

\section{Serum Alanine Aminotransferase (ALT), Triglyceride, and Total Cholesterol Measurement}

Blood was collected via cardiac puncture, centrifuged at 5,000 rpm for $15 \mathrm{~min}$, and the serum was frozen immediately. Serum ALT levels were determined by Infinity ALT (GPT) liquid stable reagent (Thermo Scientific, Middletown, VA) according to manufacturer's protocol. Serum triglyceride and total cholesterol levels were determined by Triglycerides liquid reagent set (Pointe Scientific, Inc., Canton, MI) and Cholesterol E CHOD-DAOS method (Wako, Osaka, Japan), respectively.

\section{Quantitative Real-Time Polymerase Chain Reaction (qPCR)}

The total RNAs were extracted from snap-frozen mouse liver tissues using NucleoSpin ${ }^{\circledR}$ RNA kit (Macherey-Nagel, Düren, Germany). Reverse-transcribed with High-Capacity cDNA Reverse Transcription Kit (Applied Biosystems, Foster City, CA) and $\mathrm{iTaq}^{\mathrm{TM}}$ Universal SYBR ${ }^{\circledR}$ Green Supermix (Bio-rad, Hercules, CA) were used. The sequences of mouse PCR primers are listed in Table 1. Quantitative real-time PCR was performed using CFX96 real-time PCR system (Bio-rad, Hercules, CA).
TABLE 1 | Primer sequence used for qRT-PCR.

\begin{tabular}{lll}
\hline Gene & Forward & Reverse \\
\hline 18S & AGTCCCTGCCCTTGTACACA & CGATCCGAGGGCCTCACTA \\
Fasn & GTTGGCCCAGAATCCTGTA & GTCGTCTGCCTCCAGAGC \\
Dgat1 & TCACCACACACCAATTCAGG & GACGGCTACTGGGATCTGA \\
CxCl1 & TGCACCCAAACCGAAGC & GTCAGAAGCCAGCGTTCACC \\
Ccl2 & ATTGGATCATCTTGCTGGT & CCTGCTGTTCACAGTTGCC \\
Cc/5 & CCACTTCTTCTCTGGGTGG & GTGCCCACGTCAAGGAGTAT \\
Col1a1 & TAGGCCATTGTGTATGCAGC & ACATGTCAGCTTGTGGACC \\
Acta2 & GTTCAGTGGTGCCTCTGTCA & ACTGGGACGACATGGAAAAG \\
Timp1 & AGGTGGTCTCGTTGATTCT & GTAAGGCCTGTAGCTGTGCC \\
Tgfb1 & GTGGAAATCAACGGGATCAG & ACTCCAACCCAGGTCCTTC \\
Serpine1 & TTCAGCCCTTGCTTGCTC & ACACTITACTCCGAAGTCGGT
\end{tabular}

\section{Statistical Analysis}

Differences between the two groups were compared using the Mann Whitney $U$-test or two-tailed unpaired student $t$-test. Differences between multiple groups were compared using oneway ANOVA. Statistical significance was assessed by using GraphPad Prism 5.01 software (GraphPad Software, Inc, La Jolla, Ca). $P<0.05$ were considered significant.

\section{RESULTS}

\section{The Therapeutic Effect of Recombinant HGF Protein in a Mouse Model of NASH Induced by CDAA Diet Feeding}

To investigate the therapeutic effect of recombinant HGF protein on a CDAA diet-induced mouse NASH model, wildtype C57BL/6 mice were treated with vehicle or $1 \mathrm{mg} / \mathrm{kg}$ of recombinant feline HGF protein intravenously in the last 7 days of a total of 3 weeks of CDAA diet feeding. Based on NAFLD activity scoring system, we assessed the effect of recombinant HGF on CDAA diet-induced NASH. Three weeks of CDAA diet feeding showed an evident accumulation of lipid droplets in hepatocytes, inflammatory cell infiltration, ballooned hepatocytes, and mild fibrosis in the vehicle-treated mice compared to CSAA diet feeding. The recombinant HGF treatment significantly suppressed the development of inflammation, hepatocyte ballooning, and fibrosis induced by CDAA diet feeding (Figures 1A,B). Our results indicate that recombinant HGF treatment administered on the last 7 days of CDAA diet, improved NAFLD activity score.

\section{Recombinant HGF Administration Inhibits CDAA Diet-Induced Liver Damage and Increases Hepatocyte Proliferation}

To examine the protective effect of recombinant HGF on NASHmediated hepatocyte damage, serum ALT levels were measured. Three weeks of CDAA diet feeding dramatically elevated serum ALT levels in vehicle-treated mice, whereas increased serum ALT levels by CDAA diet feeding were significantly reduced by the administration of recombinant HGF protein (Figure 2). This 

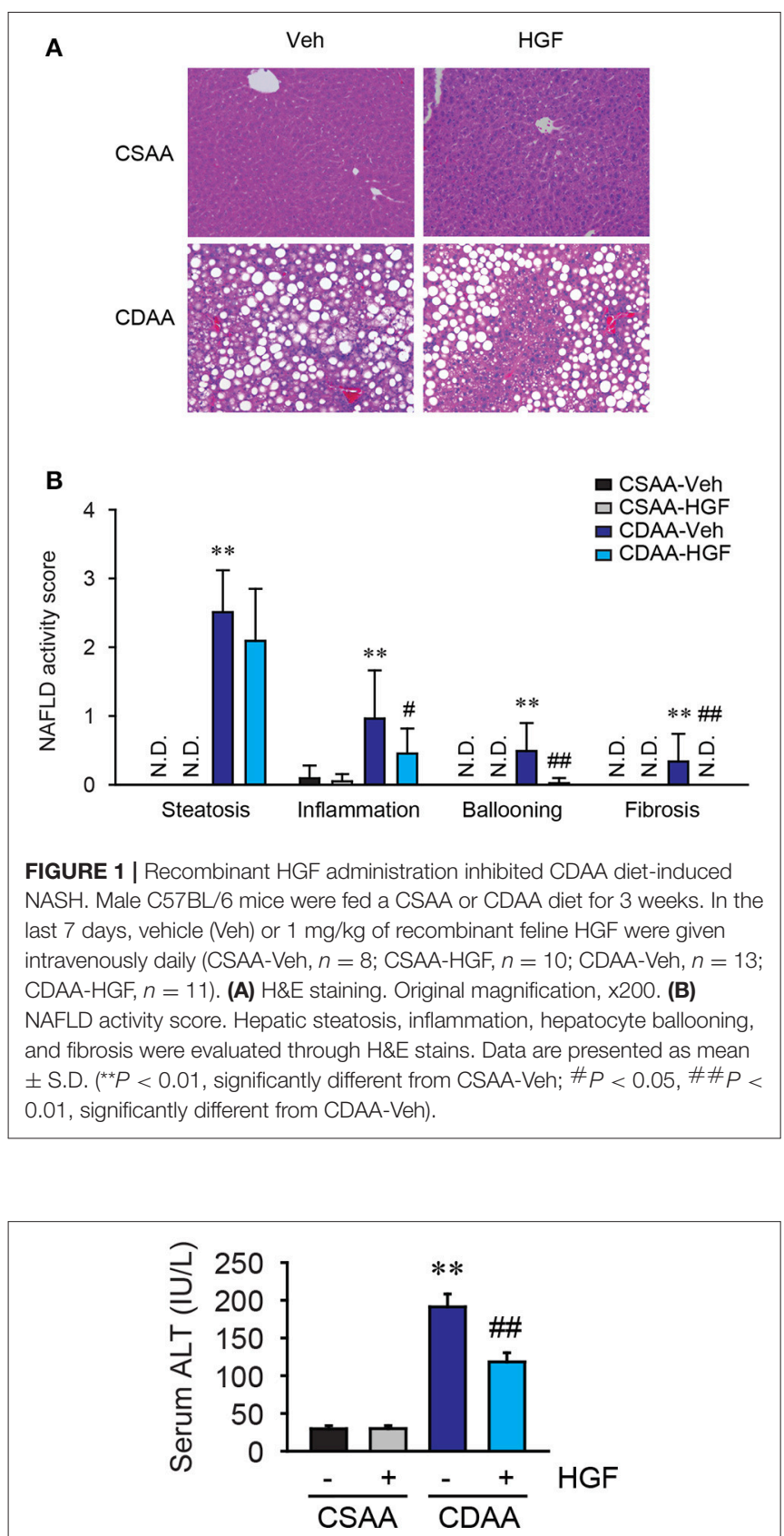

FIGURE 2 | CDAA diet-mediated liver damage was suppressed by recombinant HGF treatment. Serum ALT level. Data are presented as mean \pm SEM. $\left({ }^{\star \star} P<0.01\right.$, significantly different from CSAA-Veh; $\# \# P<0.01$, significantly different from CDAA-Veh).

result indicates that the 7 days of recombinant HGF treatment suppressed hepatocyte damage induced by the 3 weeks of CDAA diet feeding in mice.

Since HGF is a potent mitogen, we next evaluated the effect of exogenous HGF on hepatocyte proliferation. The increases in PCNA labeling were observed in both HGF-treated CSAA diet-fed and CDAA diet-fed mice (Figures 3A,B).

\section{Recombinant HGF Protein Has Anti-lipogenesis Effect in CDAA Diet-Induced Hepatic Steatosis}

In addition to the assessment of steatosis by NAFLD activity scoring system, using hematoxylin and eosin staining, hepatic steatosis was examined by oil red $\mathrm{O}$ staining. A previous report showed that H\&E steatosis assessment poorly correlated with triglyceride concentration in the tissue, whereas Oil Red O staining showed much higher sensitivity and specificity for steatosis (23).

Three weeks of CDAA diet feeding induced accumulation of large lipid droplets in hepatocytes in vehicle-treated mice but, CSAA diet feeding did not (Figures 4A,B). Serum triglyceride and total cholesterol levels were unchanged (Table 2). Seven days of daily administration of recombinant HGF protein significantly suppressed accumulation of lipid droplets in hepatocytes (Figures 4A,B). The study then assessed lipogenesisrelated gene expression. Quantitative real-time PCR analysis shows that increased Fasn and Dgatl genes by CDAA diet feeding were significantly suppressed in mice treated with recombinant HGF protein (Figure 4C). These results suggest that HGF treatment inhibits hepatic lipid accumulation through inhibiting expression of lipogenesis-related genes, such as Fasn and Dgat1.

\section{CDAA Diet-Induced Hepatic Inflammation Is Suppressed by Recombinant HGF Treatment}

Inflammatory cell infiltration is a prominent feature of NASH. Among inflammatory cells, hepatic macrophages comprising liver resident Kupffer cells, and bone marrow-derived monocytes are the significant contributor to NASH development. Liver macrophages were examined by immunohistochemical staining for F4/80, a mouse macrophage marker. While basal amounts of liver macrophages were seen in mice fed with CSAA diet, the number of liver macrophages and the foci containing macrophages were significantly augmented in mice fed with 3 weeks of CDAA diet. Interestingly, the recombinant HGF treatment reduced the amounts of liver macrophages in mice fed CDAA diet compared to vehicle treatment (Figures 5A,B). Inflammatory cell infiltration is mainly regulated by the small size $(8-10 \mathrm{kDa})$ of inflammatory cytokines, called chemokines. Increased hepatic levels of $\mathrm{Cxcl1}, \mathrm{Ccl} 2$, and $\mathrm{Ccl} 5$ induced by CDAA diet feeding were significantly reduced by the treatment of recombinant HGF (Figure 5C). Our results suggest that recombinant HGF protein has antiinflammatory effects on NASH livers that inhibit macrophage infiltration and production of chemokines which play a role in the recruitment of inflammatory cells to injured livers.

\section{Fibrogenic Response Induced by CDAA Diet Was Suppressed by Recombinant HGF Treatment}

While 3 weeks of CDAA diet feeding does not induce strong fibrillar collagen deposition, very mild collagen expression was 


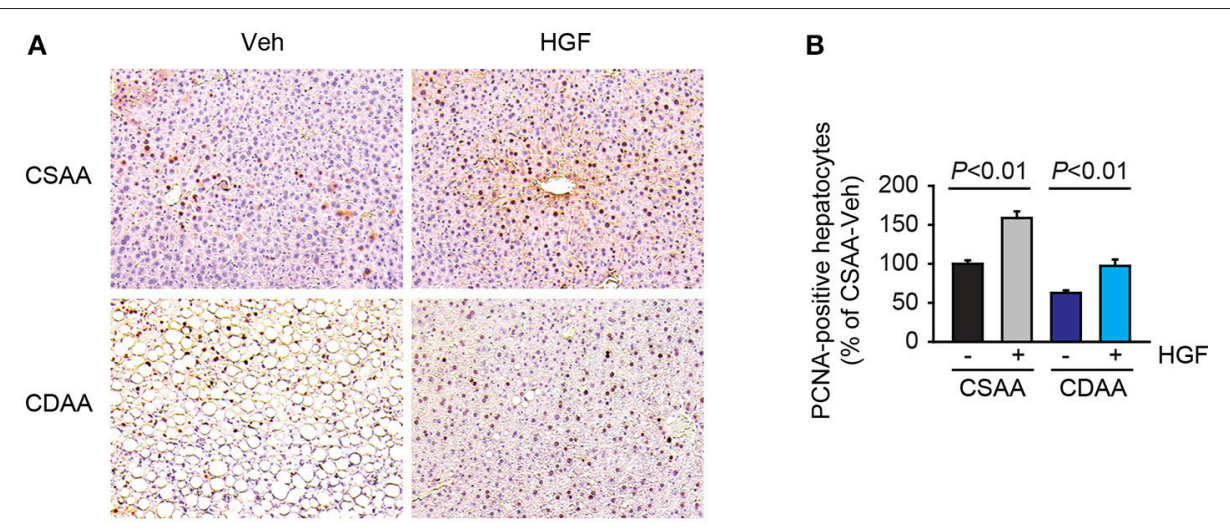

FIGURE 3 | Administration of recombinant HGF promoted hepatocyte proliferation. (A) Representative images of PCNA staining. Original magnification, x200. (B) Quantification of PCNA staining. Data are presented as mean \pm SEM.

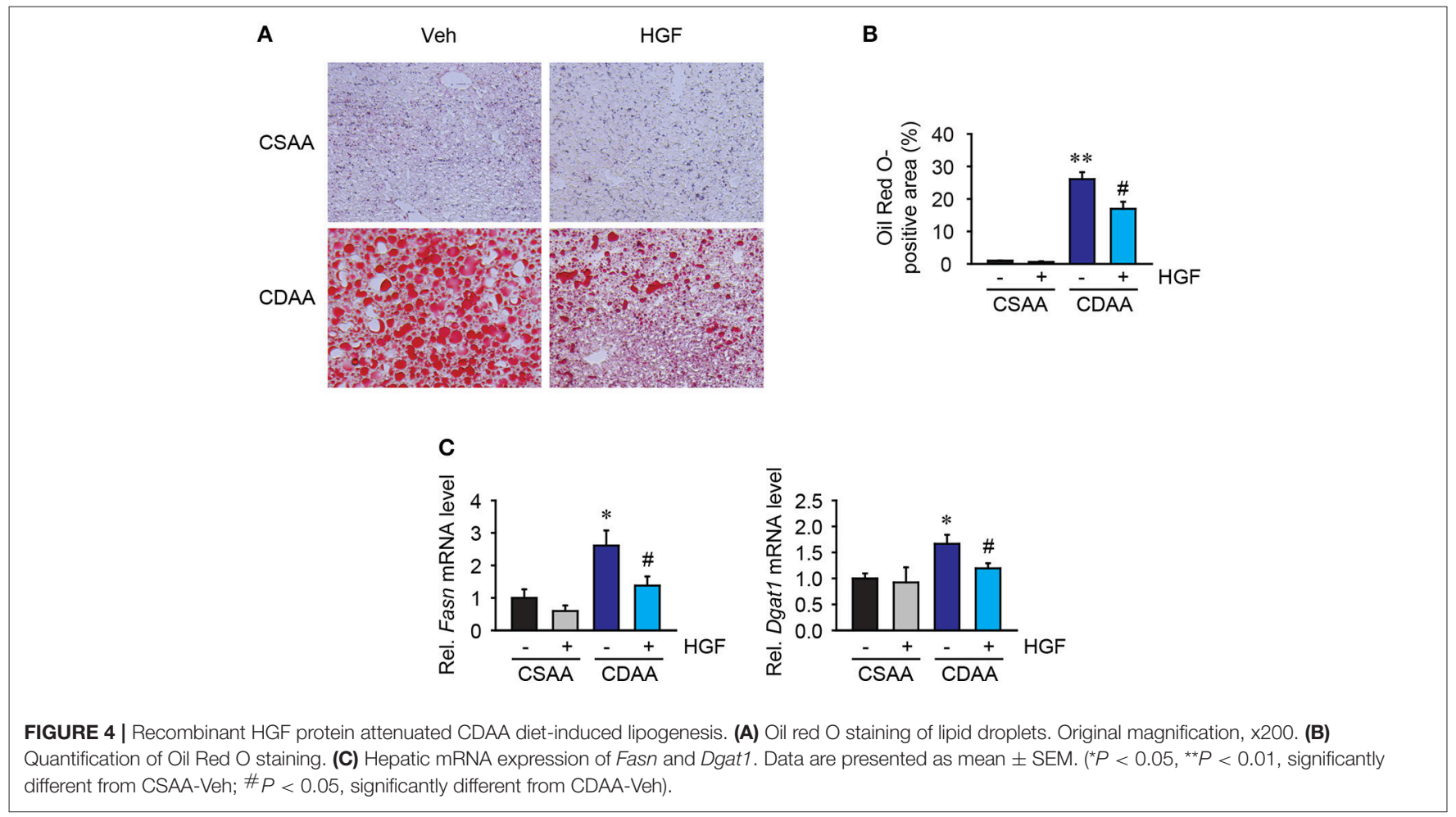

seen in liver parenchyma (Figure 6A), which was diminished by recombinant HGF treatment. Notably, there was evidence of upregulation of fibrogenic gene expression in mice with 3 weeks feeding of CDAA. In the vehicle group, CDAA diet feeding significantly upregulated Col1a1, Acta2, Timp1, Tgfb1, and Serpine1 expression in the liver (Figure 6B). On the contrary, in the recombinant HGF treatment group, upregulation of hepatic expression of fibrogenic genes Col1a1, Acta2, Timp1, Tgfb1, and Serpine1 was significantly inhibited (Figure 6B). These results suggest that recombinant HGF treatment can inhibit hepatic stellate cell activation and liver fibrosis progression in NASH.

\section{DISCUSSION}

The present study investigated the therapeutic potential of recombinant HGF protein in the development of NASH in mice. Our data demonstrated that 7 days of intravenous administration of recombinant feline HGF protein is sufficient to suppress the progression of a mouse model of NASH induced by 3 weeks of CDAA diet feeding. The HGF protein treatment significantly suppressed major pathological features of $\mathrm{NASH}$, including the accumulation of lipid droplets in hepatocytes, lipogenesis gene expression, hepatocyte ballooning, elevation of serum ALT 
TABLE 2 | The serum triglyceride and cholesterol profiles.

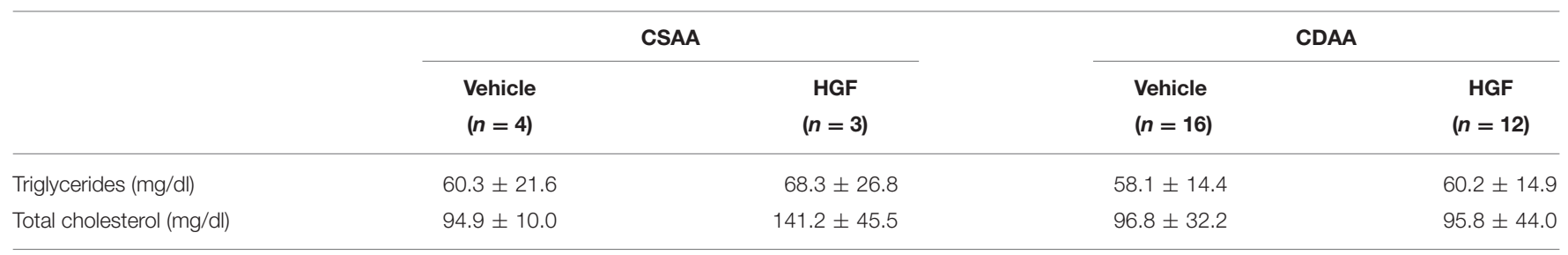

Data are presented as mean \pm S.D.

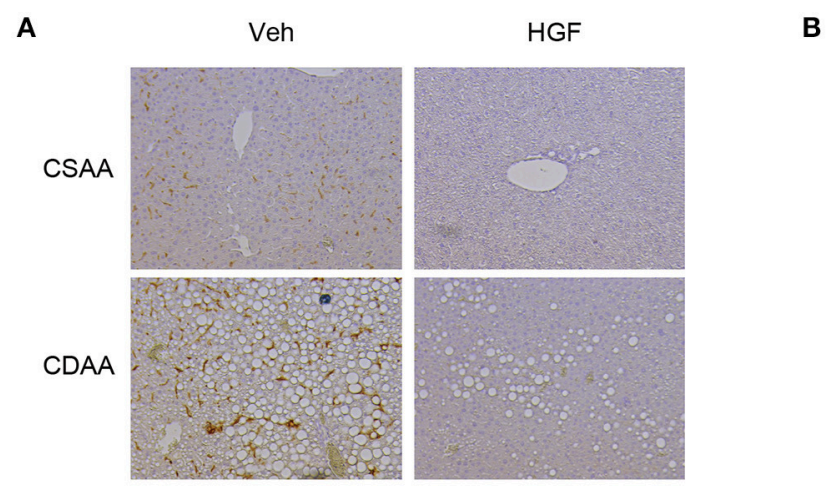

B

C

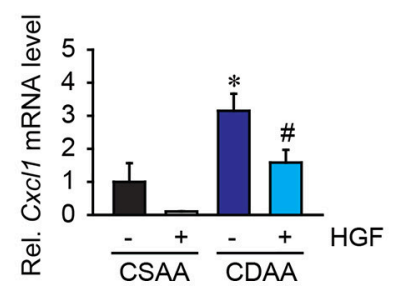

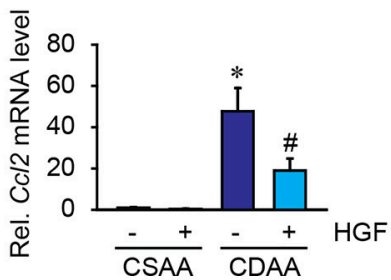

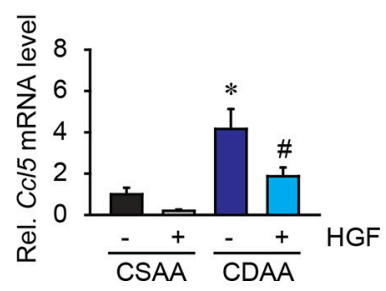

FIGURE 5 | Recombinant HGF protein had anti-inflammatory effect in CDAA diet-induced hepatic steatosis. (A) Immunohistochemistry for F4/80. Original magnification, x200. (B) Quantification of F4/80 staining. (C) Hepatic mRNA expression of Cxc/1, Ccl2, and Cc/5. Data are presented as mean \pm SEM. ( ${ }^{\star} P<0.05$, significantly different from CSAA-Veh; $\# P<0.05$, significantly different from CDAA-Veh).

levels, inflammatory cell infiltration, chemokine expression, and fibrogenic gene expression. Our study strongly suggests that administration of recombinant HGF protein can be a therapeutic option for NASH.

The physiological role of HGF and its receptor MET in the liver is well-described. HGF is initially produced and released as an inactive precursor form and is normally found in extracellular space. The major producer of HGF in the liver is hepatic stellate cell which is a precursor of myofibroblasts in the liver and contributes to the production of extracellular matrix, progressing to liver fibrosis. During massive liver damage induced by trauma, infection, toxin exposure, and inflammation, pro-HGF is proteolytically cleaved by HGF activator, urokinasetype plasminogen activator, matriptase, and so on, and becomes the biologically active form of HGF (7). The active form of HGF binds to its receptor MET, encoded by proto-oncogene $c$-met, and induces its biological actions, including hepatocyte proliferation, anti-apoptosis, wound healing, and liver tissue homeostasis. Serum HGF levels were elevated in patients with NASH compared to control group (24), proposing a compensatory mechanism responsible for hepatic regeneration. Because of the ability of HGF to induce hepatocyte proliferation and liver regeneration, HGF has been suggested to be a potential therapeutic agent for liver cirrhosis (25).

Hepatocyte damage and liver fibrosis are the key pathological features in NASH. Even though serum HGF levels were increased in NASH patients, it is unclear whether the active form of HGF was also increased in NASH patients. In addition, HGF receptor, MET was underexpressed in human NAFLD livers compared to control livers (26). Our data demonstrated that exogenous HGF treatment suppressed CDAA diet-induced hepatocyte damage and fibrogenic response. Previous studies also demonstrated the protective role of the HGF-MET pathway in liver injury and fibrosis. Exogenous administration of recombinant HGF protein suppresses lipopolysaccharide-induced or Fas-mediated mouse models of fulminant hepatitis $(12,27,28)$. In Fas-mediated liver injury model, HGF induces Mcl-1 expression and activation of Akt pathway, resulting in inhibition of hepatocyte apoptosis 

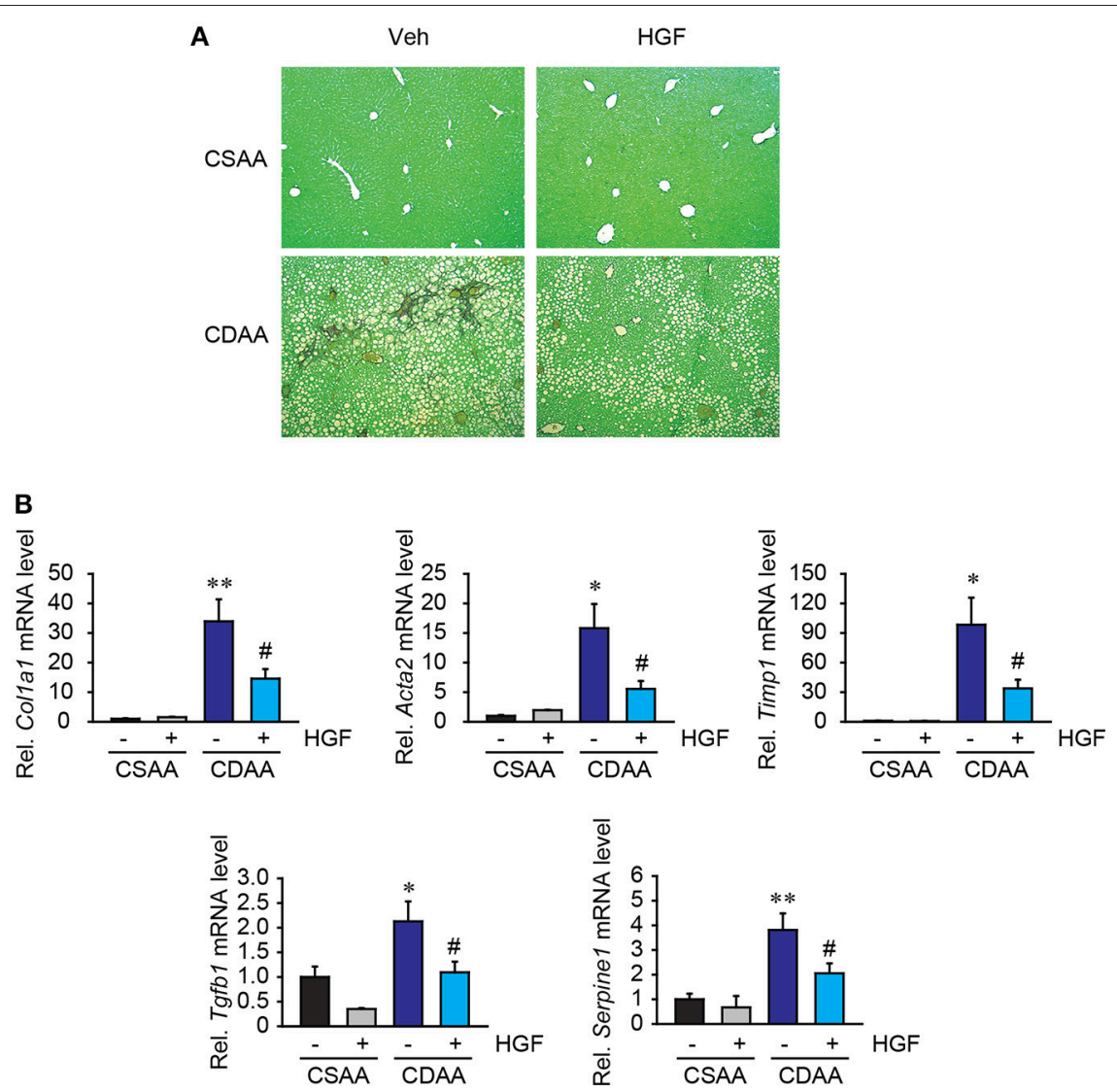

FIGURE 6 | Recombinant HGF ameliorated CDAA diet-induced fibrogenic response. (A) Sirius Red staining. Original magnification, x100. (B) Hepatic mRNA expression of Col1a1, Acta2, Timp1, Tgfb1, and Serpine1. Data are presented as mean \pm SEM. $\left({ }^{\star} P<0.05\right.$, ${ }^{\star \star} P<0.01$, significantly different from CSAA-Veh; $\# P<$ 0.05 , significantly different from CDAA-Veh).

(28). In addition, HGF stimulates hepatocyte proliferation (Figure 3), which is favorable to the liver repair process. Since one of the major functions of HGF is the induction of matrix metalloproteases (MMPs), such as membrane type 1-MMP and MMP9 to degrade the extracellular matrix, a number of reports demonstrated the therapeutic effect of HGF in liver fibrosis $(10,11,25,29)$. HGF is also known to have an anti-inflammatory property. HGF treatment reduced expression of TNF- $\alpha$, CCL2, and IL-6 in mouse culture macrophages, in which induction of anti-inflammatory heme oxygenase-1 and IL-10 are partly involved $(30,31)$. These reports are consistent with our data showing that recombinant HGF treatment inhibited NASHmediated macrophage infiltration and chemokine expression in the liver.

The protective effect of HGF against lipids in hepatocytes has previously been reported. Recombinant HGF treatment reduced intracellular lipid content, likely by accelerating lipid secretion in hepatocytes through activation of microsomal triglyceride transfer protein and apolipoprotein B (32). Moreover, recombinant HGF treatment inhibited cholesterol overload-mediated hepatocyte lipotoxicity by suppressing production of reactive oxygen species (33). An animal study using hepatocyte-specific c-met-deleted mice showed exacerbation of a mouse model of NASH induced by methioninecholine deficient diet (13). All of these reports suggest both exogenous and endogenous HGF-MET system play a critical role in the protection of NAFLD development. Blockade of mineralocorticoid receptor signaling attenuates hepatic steatosis and insulin resistance in a mouse model of obesity. Interestingly, the underlying mechanism of attenuated hepatic steatosis by blocking mineralocorticoid receptor is mediated through the HGF-MET pathway (34). Our data demonstrated that exogenous HGF treatment reduced hepatic steatosis along with reduction of lipogenesis genes, but it appears that the protective mechanism of HGF is not mediated through modulation of lipid degradation pathway as our data did not show any changes in the expression of $\beta$-oxidation-related genes (data not shown).

Obesity and fatty liver disease have been often observed in cats and dogs $(35,36)$. Like in humans, obesity is closely associated with metabolic syndrome. High-fat diet feeding altered the composition of fatty acid in liver tissue and serum (37). The stearic acid-rich high fat diet promoted hepatic lipogenesis in the feline liver (37). Feline hepatic lipidosis, also known as feline fatty liver syndrome, is the most diagnosed liver disease in cats 
(38). This disease is life-threatening without treatment, but the prognosis would be favored in cases of intensive treatment and adequate therapy. The causes and pathogenic mechanisms are scarcely known. Since our data clearly showed that exogenous HGF treatment efficiently reduced the fat content in the liver and inhibited CDAA diet-induced lipogenesis in mice, further evaluation in large animals may be needed to determine the efficacy and safety of HGF in fatty liver disease.

The MET overexpression and activation play a crucial role in cancer cell proliferation, migration, invasion, and metastasis. This signaling also contributes to drug resistance in tumor microenvironment (39). In HCC, MET is overexpressed (40), but some reports showed HGF is underexpressed (41-45). The effect of HGF on hepatocarcinogenesis is controversial. HGF treatment suppressed HCC cell growth in vitro in some reports $(46,47)$. Exogenous HGF administration or HGF transgenic mouse model showed both pro- and anti-tumorigenic effects of HGF (48-56). Nakanishi et al. tested the long-term effect of exogenous HGF to the NASH mouse model on the occurrence of HCC (56). According to their results, treatment with recombinant human HGF did not increase the overall frequency of HCC. We suggest that NAFLD patients or animals with cancer or individuals with the potential to have cancer are not appropriate for HGF treatment.

Besides the beneficial role of HGF in the liver, HGF is also involved in tissue regeneration, protection, and homeostasis in the kidney and neurons (57). Moreover, HGF is a potent inducer of angiogenesis and has a protective role in the development of cardiovascular diseases through modulation of atherosclerosis (58). Given that the leading cause of death by NASH is cardiovascular diseases (59), targeting of the HGF-MET pathway should have beneficial effects on both liver and heart. Therefore, it is worth considering HGF therapy for NAFLD, but the careful

\section{REFERENCES}

1. Day CP. Non-alcoholic fatty liver disease: a massive problem. Clin Med (Lond). (2011) 11:176-8.

2. Kneeman JM, Misdraji J, Corey KE. Secondary causes of nonalcoholic fatty liver disease. Therap Adv Gastroenterol. (2012) 5:199-207. doi: 10.1177/1756283X11430859

3. Sanyal AJ, Brunt EM, Kleiner DE, Kowdley KV, Chalasani N, Lavine JE, et al. Endpoints and clinical trial design for nonalcoholic steatohepatitis. Hepatology (2011) 54:344-53. doi: 10.1002/hep.24376

4. Tiniakos DG, Vos MB, Brunt EM. Nonalcoholic fatty liver disease: pathology and pathogenesis. Annu Rev Pathol. (2010) 5:145-71. doi: 10.1146/annurev-pathol-121808-102132

5. Chedid MF. Nonalcoholic steatohepatitis: the second leading indication for liver transplantation in the USA. Dig Dis Sci. (2017) 62:2621-2. doi: 10.1007/s10620-017-4724-6

6. Webb CB. Hepatic lipidosis: clinical review drawn from collective effort. $J$ Feline Med Surg. (2018) 20:217-27. doi: 10.1177/1098612X18758591

7. Nakamura T, Sakai K, Nakamura T, Matsumoto K. Hepatocyte growth factor twenty years on: much more than a growth factor. J Gastroenterol Hepatol. (2011) 26:188-202. doi: 10.1111/j.1440-1746.2010.06549.x

8. Tahara M, Matsumoto K, Nukiwa T, Nakamura T. Hepatocyte growth factor leads to recovery from alcohol-induced fatty liver in rats. J Clin Invest. (1999) 103:313-20. doi: 10.1172/JCI4433 selection of patients or animals may have to be considered due to the capacity of HGF to promote cancer progression.

In summary, the present study demonstrated that exogenous administration of recombinant feline HGF protein has potential to mitigate the development of NASH. Although there is a concern about the role of HGF played in cancer progression, it should be considered for those individuals with low risk of developing HCC (e.g., early stage of NASH fibrosis without cirrhosis). Given that HGF can suppress the risk of cardiovascular disease which is the leading cause of death in NASH patients, exogenous HGF administration may be a favorable option for the treatment of NAFLD.

\section{AUTHOR CONTRIBUTIONS}

YMY, MF, MK, and ES contributed conception and design of the study. YMY, MF, ZW, and FM performed experiments, analysis and interpretation of the data, and statistical analysis. YMY and ES wrote the first draft of the manuscript. All authors contributed to manuscript revision, read and approved the submitted version.

\section{FUNDING}

This study was supported by the research grant from Nippon Zenyaku Kogyo Co Ltd. The funder provided support for author MF to participate in UCD postdoctoral fellowship program and research support to UCSD. This study was also supported by NIH grant R01DK085252 (ES), R21AA025841 (ES), T32HL134637 (YMY). American Liver Foundation Irwin M. Arias, MD Postdoctoral Research Fellowship (YMY). AASLD The Leonard B. Seeff Award for Outstanding Research by Young Investigator (YMY) and Winnick Research award from Cedars-Sinai Medical Center (ES).
9. Sakakura Y, Kaibori M, Oda M, Okumura T, Kwon AH, Kamiyama Y. Recombinant human hepatocyte growth factor protects the liver against hepatic ischemia and reperfusion injury in rats. J Surg Res. (2000) 92:261-6. doi: $10.1006 /$ jsre. 2000.5913

10. Kusumoto K, Ido A, Moriuchi A, Katsura T, Kim I, Takahama Y, et al. Repeated intravenous injection of recombinant human hepatocyte growth factor ameliorates liver cirrhosis but causes albuminuria in rats. Int J Mol Med. (2006) 17:503-9.

11. Matsuda Y, Matsumoto K, Yamada A, Ichida T, Asakura $H$, Komoriya Y, et al. Preventive and therapeutic effects in rats of hepatocyte growth factor infusion on liver fibrosis/cirrhosis. Hepatology (1997) 26:81-9. doi: 10.1053/jhep.1997.v26.pm00092 14455

12. Kosai K, Matsumoto K, Funakoshi H, Nakamura T. Hepatocyte growth factor prevents endotoxin-induced lethal hepatic failure in mice. Hepatology (1999) 30:151-9. doi: 10.1002/hep.510300102

13. Kroy DC, Schumacher F, Ramadori P, Hatting M, Bergheim I, Gassler N, et al. Hepatocyte specific deletion of c-Met leads to the development of severe non-alcoholic steatohepatitis in mice. J Hepatol. (2014) 61:883-90. doi: 10.1016/j.jhep.2014.05.019

14. Tojima H, Kakizaki S, Kosone T, Horiguchi N, Yamazaki Y, Sato K, et al. Hepatocyte growth factor overexpression ameliorates liver inflammation and fibrosis in a mouse model of nonalcoholic steatohepatitis. Hepatol Int. (2012) 6:620-30. doi: 10.1007/s12072-011-9301-z 
15. Kiyama S, Yamada T, Iwata H, Sekino T, Matsuo H, Yoshida N, et al. Reduction of fibrosis in a rat model of non-alcoholic steatohepatitis cirrhosis by human HGF gene transfection using electroporation. J Gastroenterol Hepatol. (2008) 23:e471-6. doi: 10.1111/j.1440-1746.2007.05111.x

16. Yang L, Miura K, Zhang B, Matsushita H, Yang YM, Liang S, et al. TRIF differentially regulates hepatic steatosis and inflammation/fibrosis in mice. Cell Mol Gastroenterol Hepatol. (2017) 3:469-83. doi: 10.1016/j.jcmgh.2016.12.004

17. Miura K, Kodama Y, Inokuchi S, Schnabl B, Aoyama T, Ohnishi $\mathrm{H}$, et al. Toll-like receptor 9 promotes steatohepatitis by induction of interleukin-1 $\beta$ in mice. Gastroenterology (2010) 139:323-34.e7. doi: 10.1053/j.gastro.2010.03.052

18. Kruitwagen HS, Arends B, Spee B, Brinkhof B, van den Ingh TS, Rutten VP, et al. Recombinant hepatocyte growth factor treatment in a canine model of congenital liver hypoplasia. Liver Int. (2011) 31:940-9. doi: 10.1111/j.1478-3231.2011.02513.x

19. Kobayashi Y, Nakamura N, Ishizaka T, Masuda K, Ohno K, Tsujimoto H. Molecular cloning of feline hepatocyte growth factor (HGF) cDNA. J Vet Med Sci. (2001) 63:211-4.

20. Kleiner DE, Brunt EM, Van Natta M, Behling C, Contos MJ, Cummings OW, et al. Design and validation of a histological scoring system for nonalcoholic fatty liver disease. Hepatology (2005) 41:1313-21. doi: 10.1002/hep.20701

21. Song IJ, Yang YM, Inokuchi-Shimizu S, Roh YS, Yang L, Seki E. The contribution of toll-like receptor signaling to the development of liver fibrosis and cancer in hepatocyte-specific TAK1-deleted mice. Int J Cancer. (2018) 142:81-91. doi: 10.1002/ijc.31029

22. Inokuchi S, Aoyama T, Miura K, Osterreicher $\mathrm{CH}$, Kodama Y, Miyai K, et al. Disruption of TAK1 in hepatocytes causes hepatic injury, inflammation, fibrosis, and carcinogenesis. Proc Natl Acad Sci USA. (2010) 107:844-9. doi: 10.1073/pnas.0909781107

23. Levene AP, Kudo H, Armstrong MJ, Thursz MR, Gedroyc WM, Anstee QM, et al. Quantifying hepatic steatosis-more than meets the eye. Histopathology (2012) 60:971-81. doi: 10.1111/j.1365-2559.2012.04193.x

24. Agrawal RP, Sheroan V, Ola V, Sulemani AA, Singh N, Sirohi P, et al. Hepatocyte growth factor, adiponectin and hepatic histopathology in nonalcoholic steatohepatitis. J Assoc Physicians India. (2013) 61:789-92.

25. Ueki T, Kaneda Y, Tsutsui H, Nakanishi K, Sawa Y, Morishita R, et al. Hepatocyte growth factor gene therapy of liver cirrhosis in rats. Nat Med. (1999) 5:226-30. doi: 10.1038/5593

26. Zou C, Ma J, Wang X, Guo L, Zhu Z, Stoops J, et al. Lack of Fas antagonism by Met in human fatty liver disease. Nat Med. (2007) 13:1078-85. doi: $10.1038 / \mathrm{nm} 1625$

27. Kosai K, Matsumoto K, Nagata S, Tsujimoto Y, Nakamura T. Abrogation of Fas-induced fulminant hepatic failure in mice by hepatocyte growth factor. Biochem Biophys Res Commun. (1998) 244:683-90.

28. Schulze-Bergkamen H, Brenner D, Krueger A, Suess D, Fas SC, Frey CR, et al. Hepatocyte growth factor induces Mcl-1 in primary human hepatocytes and inhibits CD95-mediated apoptosis via Akt. Hepatology (2004) 39:645-54. doi: 10.1002/hep.20138

29. Xia JL, Dai C, Michalopoulos GK, Liu Y. Hepatocyte growth factor attenuates liver fibrosis induced by bile duct ligation. Am J Pathol. (2006) 168:1500-12. doi: 10.2353/ajpath.2006.050747

30. Kusunoki H, Taniyama Y, Otsu R, Rakugi H, Morishita R. Anti-inflammatory effects of hepatocyte growth factor on the vicious cycle of macrophages and adipocytes. Hypertens Res. (2014) 37:500-6. doi: 10.1038/hr.2014.41

31. Kamimoto M, Mizuno S, Nakamura T. Reciprocal regulation of IL-6 and IL10 balance by HGF via recruitment of heme oxygenase- 1 in macrophages for attenuation of liver injury in a mouse model of endotoxemia. Int J Mol Med. (2009) 24:161-70.

32. Kosone T, Takagi H, Horiguchi N, Ariyama Y, Otsuka T, Sohara N, et al. HGF ameliorates a high-fat diet-induced fatty liver. Am J Physiol Gastrointest Liver Physiol. (2007) 293:G204-10. doi: 10.1152/ajpgi.00021.2007

33. Dominguez-Perez M, Nuno-Lambarri N, Clavijo-Cornejo D, Luna-Lopez A, Souza V, Bucio L, et al. Hepatocyte growth factor reduces free cholesterolmediated lipotoxicity in primary hepatocytes by countering oxidative stress. Oxid Med Cell Longev. (2016) 2016:7960386. doi: 10.1155/2016/7960386

34. Zhang YY, Li C, Yao GF, Du LJ, Liu Y, Zheng XJ, et al. Deletion of macrophage mineralocorticoid receptor protects hepatic steatosis and insulin resistance through ERalpha/HGF/Met pathway. Diabetes (2017) 66:1535-47. doi: $10.2337 / \mathrm{db} 16-1354$

35. German AJ. The growing problem of obesity in dogs and cats. J Nutr. (2006) 136:1940S-6S. doi: 10.1093/jn/136.7.1940S

36. Roth L. Comparison of liver cytology and biopsy diagnoses in dogs and cats: 56 cases. Vet Clin Pathol. (2001) 30:35-8.

37. Fujiwara M, Mori N, Sato T, Tazaki H, Ishikawa S, Yamamoto I, et al. Changes in fatty acid composition in tissue and serum of obese cats fed a high fat diet. BMC Vet Res. (2015) 11:200. doi: 10.1186/s12917-015-0519-1

38. Kuzi S, Segev G, Kedar S, Yas E, Aroch I. Prognostic markers in feline hepatic lipidosis: a retrospective study of 71 cats. Vet Rec. (2017) 181:512. doi: $10.1136 / v r .104252$

39. Imamura $\mathrm{R}$, Matsumoto K. Hepatocyte growth factor in physiology and infectious diseases. Cytokine (2017) 98:97-106. doi: 10.1016/j.cyto.2016.12.025

40. You H, Ding W, Dang H, Jiang Y, Rountree CB. c-Met represents a potential therapeutic target for personalized treatment in hepatocellular carcinoma. Hepatology (2011) 54:879-89. doi: 10.1002/hep.24450

41. Selden C, Farnaud S, Ding SF, Habib N, Foster C, Hodgson HJ. Expression of hepatocyte growth factor mRNA, and c-met mRNA (hepatocyte growth factor receptor) in human liver tumours. J Hepatol. (1994) 21:227-34.

42. Noguchi O, Enomoto N, Ikeda T, Kobayashi F, Marumo F, Sato C. Gene expressions of c-met and hepatocyte growth factor in chronic liver disease and hepatocellular carcinoma. J Hepatol. (1996) 24:286-92.

43. Kiss A, Wang NJ, Xie JP, Thorgeirsson SS. Analysis of transforming growth factor (TGF)-alpha/epidermal growth factor receptor, hepatocyte growth Factor/c-met,TGF-beta receptor type II, and p53 expression in human hepatocellular carcinomas. Clin Cancer Res. (1997) 3:1059-66.

44. Tavian D, De Petro G, Benetti A, Portolani N, Giulini SM, Barlati S. u-PA and c-MET mRNA expression is co-ordinately enhanced while hepatocyte growth factor mRNA is down-regulated in human hepatocellular carcinoma. Int $J$ Cancer. (2000) 87:644-9.

45. Daveau M, Scotte M, Francois A, Coulouarn C, Ros G, Tallet Y, et al. Hepatocyte growth factor, transforming growth factor alpha, and their receptors as combined markers of prognosis in hepatocellular carcinoma. $\mathrm{Mol}$ Carcinog. (2003) 36:130-41. doi: 10.1002/mc.10103

46. Tajima H, Matsumoto K, Nakamura T. Hepatocyte growth factor has potent anti-proliferative activity in various tumor cell lines. FEBS Lett. (1991) 291:229-32.

47. Shiota G, Rhoads DB, Wang TC, Nakamura T, Schmidt EV. Hepatocyte growth factor inhibits growth of hepatocellular carcinoma cells. Proc Natl Acad Sci USA. (1992) 89:373-7.

48. Liu ML, Mars WM, Michalopoulos GK. Hepatocyte growth factor inhibits cell proliferation in vivo of rat hepatocellular carcinomas induced by diethylnitrosamine. Carcinogenesis (1995) 16:841-3.

49. Yaono M, Hasegawa R, Mizoguchi Y, Futakuchi M, Nakamura T, Ito $\mathrm{N}$, et al. Hepatocyte growth factor enhancement of preneoplastic hepatic foci development in rats treated with diethylnitrosamine and N-ethyl-N-hydroxyethylnitrosamine. Jpn J Cancer Res. (1995) 86:718-23.

50. Ogasawara H, Hiramoto J, Takahashi M, Shirahama K, Furusaka A, Hiyane S, et al. Hepatocyte growth factor stimulates DNA synthesis in rat preneoplastic hepatocytes but not in liver carcinoma cells. Gastroenterology (1998) 114:77581.

51. Sakata H, Takayama H, Sharp R, Rubin JS, Merlino G, LaRochelle WJ. Hepatocyte growth factor/scatter factor overexpression induces growth, abnormal development, and tumor formation in transgenic mouse livers. Cell Growth Differ. (1996) 7:1513-23.

52. Horiguchi N, Takayama H, Toyoda M, Otsuka T, Fukusato T, Merlino G, et al. Hepatocyte growth factor promotes hepatocarcinogenesis through c-Met autocrine activation and enhanced angiogenesis in transgenic mice treated with diethylnitrosamine. Oncogene (2002) 21:1791-9. doi: $10.1038 /$ sj.onc. 1205248

53. Shiota G, Wang TC, Nakamura T, Schmidt EV. Hepatocyte growth factor in transgenic mice: effects on hepatocyte growth, liver regeneration and gene expression. Hepatology (1994) 19:962-72. 
54. Santoni-Rugiu E, Preisegger KH, Kiss A, Audolfsson T, Shiota G, Schmidt $\mathrm{EV}$, et al. Inhibition of neoplastic development in the liver by hepatocyte growth factor in a transgenic mouse model. Proc Natl Acad Sci USA. (1996) 93:9577-82.

55. Goyal L, Muzumdar MD, Zhu AX. Targeting the HGF/c-MET pathway in hepatocellular carcinoma. Clin Cancer Res. (2013) 19:2310-8. doi: 10.1158/1078-0432.CCR-12-2791

56. Nakanishi C, Moriuchi A, Ido A, Numata M, Kim ID, Kusumoto K, et al. Effect of hepatocyte growth factor on endogenous hepatocarcinogenesis in rats fed a choline-deficient L-amino acid-defined diet. Oncol Rep. (2006) 16:25-31.

57. Matsumoto K, Funakoshi H, Takahashi H, Sakai K. HGF-Met pathway in regeneration and drug discovery. Biomedicines (2014) 2:275-300. doi: 10.3390/biomedicines2040275

58. Madonna R, Cevik C, Nasser M, De Caterina R. Hepatocyte growth factor: molecular biomarker and player in cardioprotection and cardiovascular regeneration. Thromb Haemost. (2012) 107:656-61. doi: 10.1160/TH11-10-0711
59. Adams LA, Anstee QM. A fatty liver leads to a broken heart? J Hepatol. (2016) 65:14-6. doi: 10.1016/j.jhep.2016.03.012

Conflict of Interest Statement: The authors have the following interests. The study was supported by Nippon Zenyaku Kogyo Co Ltd, the employer of MF.

The remaining authors declare that the research was conducted in the absence of any commercial or financial relationships that could be construed as a potential conflict of interest.

Copyright (C) 2018 Yang, Fukui, Wang, Miao, Karriker and Seki. This is an openaccess article distributed under the terms of the Creative Commons Attribution License (CC BY). The use, distribution or reproduction in other forums is permitted, provided the original author(s) and the copyright owner(s) are credited and that the original publication in this journal is cited, in accordance with accepted academic practice. No use, distribution or reproduction is permitted which does not comply with these terms. 